\title{
Enfisema y tabaquismo en pacientes con exposición ocupacional al asbesto
}

\author{
Miguel Brun Otero \\ Complexo Hospitalario Universitario de Ferrol. Ferrol (La Coruña)
}

El artículo "Enfisema y tabaquismo en pacientes con exposición ocupacional al asbesto" constituye en nuestro conocimiento el primer artículo publicado en España de estas características. También en la literatura médica hay escasos artículos publicados que hayan estudiado la relación del enfisema y el tabaquismo en pacientes con antecedente de exposición ocupacional al asbesto $^{1-5}$, lo cual proporciona mayor importancia al presente trabajo. De todos estos artículos el que ha estudiado específicamente la relación entre enfisema y exposición al asbesto es el trabajo de Huuskonen del año 2004², si bien, él lo hace con trabajadores de la construcción, partiendo de 2 premisas: una, que el enfisema se ha relacionado con otras patologías asociadas a exposiciones ocupacionales, como es la silicosis o la neumoconiosis de los mineros del carbón, y, dos, que los trabajadores de la construcción se exponen a un ambiente con numerosos agentes que tienen efectos perjudiciales sobre el pulmón, uno de los cuales podría ser el enfisema. Lo primero que hay que destacar del presente trabajo es que dentro de este grupo de trabajadores $y$, como cabía esperar, el enfisema se asoció a la historia de tabaquismo, de hecho, dentro del grupo de los nunca fumadores no se objetivó enfisema. Esto concuerda con otros trabajos publicados. El porcentaje de enfisema dentro del grupo de los fumadores y exfumadores es elevado (12 trabajadores con enfisema dentro de los 35 fumadores y exfumadores, es decir, un $34,28 \%)$. Esta cifra tan elevada puede ser explicada por el hecho de que los trabajadores objetos del estudio provienen de la construcción naval, la cual ha sido junto con la extracción del mineral, manipulación de cemento que contenga asbesto, también conocido como fibrocemento o uralita, y aislamientos, los trabajos donde ha habido una mayor exposición al amianto6-11. El trabajo de Huuskonen precisamente encontró asociación entre la exposición intensa al amianto y la presencia de enfisema. También el estudio japonés de $2018^{5}$ encuentra correlación entre el grado de tabaquismo, la presencia de cambios de fibrosis y enfisema y el derrame pleural con el hallazgo de cáncer de pulmón en una población de más de 2000 trabajadores a los que se hizo Tomografía Computarizada (TC) de baja dosis para detectar patología ocasionada por el asbesto. Otro hallazgo de interés en el presente estudio es la correlación entre grado de tabaquismo y la presencia de enfisema.

Como crítica al presente estudio se echa de menos una descripción así como detallar el número de trabajadores afectados por cada una de las patologías relacionadas con la exposición al amianto o asbesto, a saber: placas pleurales, engrosamiento pleural difuso, fibrosis pulmonar por asbesto o asbestosis, derrame pleural, o presencia de patología maligna como el cáncer de pulmón o el mesotelioma ${ }^{12}$. Si bien, la muestra es pequeña, tal y como reconocen los autores, y es, por tanto, dificil valorar la asociación de enfisema con algún otro hallazgo radiológico, hubiera sido interesante conocer la incidencia de la enfermedad por asbesto y su proporción en este grupo de trabajadores.

Teniendo en cuenta que el enfisema constituye por sí solo un factor de riesgo para el cáncer de pulmón, y en este grupo su incidencia es eleva- 
"en la literatura médica hay escasos artículos publicados que hayan estudiado la relación del enfisema y el tabaquismo en pacientes con antecedente de exposición ocupacional al asbesto 1-5, lo cual proporciona mayor importancia al presente trabajo"

da, el importante grado de tabaquismo de esta serie de trabajadores, y que el asbesto está reconocido como carcinógeno del grupo IA para cáncer de pulmón por la IARC (International Agency Research for Cancer), organismo dependiente de la OMS (Organización Mundial de la Salud), esto es, se sabe que produce cáncer de pulmón en humanos, además de haberse descrito un efecto sinérgico con el tabaquismo, no sería descabellado pensar que este grupo de trabajadores podrían ser candidatos a un programa de screening de cáncer de pulmón con TC de baja dosis tal como propone el estudio NSLT'13. No obstante, este punto debería ser confirmado mediante otro tipo de estudio.

La importancia, por tanto, del presente trabajo es el ser el primero de estas características publicado en nuestro país y, tal como concluyen los autores, el haber demostrado una relación entre enfisema paraseptal y tabaquismo acumulado en un grupo de trabajadores expuestos intensamente al asbesto, como lo son los trabajadores de la industria naval.

\section{Bibliografía}

1. Remy-Jardin M, Sobaszek A, Duhamel A, Mastora I, Zanetti C, Remy J. Asbestosrelated pleuropulmonary diseases: evaluation with low-dose four-detector row spiral CT. Radiology. 2004;233:182-90.

2. Huuskonen 0 , Kivisaari L, Zitting A, Kaleva S, Vehmas T. Emphysema findings associated with heavy asbestos-exposure in high resolution computed tomography of finnish construction workers. J Occup Health 2004 Jul;46(4):266-71.

3. Vierikko $T$, Järvenpää $R$, Toivio $P$, Uitti J, Oksa P, Lindholm T, et al. Clinical and HRCT screening of heavily asbestos-exposed workers. Int Arch Occup Environ Health. 2010;83:47-54.

4. Vehmas T, Oksa P. Chest HRCT signs predict deaths in long-term follow-up among asbestos exposed workers. Eur J Radiol. 2014 Oct;83 (10):1983-7.

5. Katoa K, Gembab K, Ashizawac K, Arakawad H, Hondae S, Noguchif N, Hondac S, Fujimotog N, Kishimoto T. Low-dose chest computed tomography screening of subjects exposed to asbestos. Eur J Radiol. 2018 Apr; 101:124-128.

6. Soulat JM, Lauque D, Esquirol Y, Déprés M, Giron J, Claudel R, et al. High-resolution computed tomography abnormalities in ex-insulators annually exposed to asbestos dust. Am J Ind Med. 1999 Dec;36(6):593-601.

7. Tagnon I, Blot WJ, Stroube RB, Day NE, Morris LE, Peace BB, et al. Mesothelioma associated with the shipbuilding industry in coastal Virginia. Cancer Res. 1980 Dec;40(11):3875-9.

8. McElvenny DM, Darnton AJ, Price MJ, Hodgson JT. Mesothelioma mortality in Great Britain from 1968 to 2001. Occup Med (Lond). 2005 Mar 1;55(2):79-87.

9. Darnton AJ, McElvenny DM, Hodgson JT. Estimating the number of asbestos-related lung cancer deaths in Great Britain from 1980 to 2000. Ann Occup Hyg. 2006 Jan 1;50(1):29-38.

10. Jemal A, Grauman D, Devesa S. Recent geographic patterns of lung cancer and mesothelioma mortality rates in 49 shipyard counties in the United States, 1970-94. Am J Ind Med. 2000 May;37(5):512-21.

11. Van Oyen SC, Peters S, Alfonso H, Fritschi L, de Klerk NH, Reid A, et al. Development of a Job-Exposure Matrix (AsbJEM) to Estimate Occupational Exposure to Asbestos in Australia. Ann Occup Hyg. $2015 \mathrm{Jul} ; 59(6): 737-48$.

12. Diego Roza C, Cruz Carmona MJ, Fernández Álvarez R, Ferrer Sancho J, Marín Martínez B, Martínez González C, Rodríguez Portal JA, Romero Valero F, Villena Garrido V. Recomendaciones sobre el diagnóstico y manejo de la enfermedad pleural y pulmonar por asbesto. Arch Bronconeumol 2017;53:437-42.

13. D.R. Aberle, A.M. Adams, C.D. Berg, W.C. Black, J.D. Clapp, R.M. Fagerstrom, National Lung Screening Trial Research Team. Reduced lung-cancer mortality with low-dose computed tomographic screening. N Engl J Med., 365 (2011), pp. 395-409. 